\title{
Mutational profiles of persistent/recurrent laryngeal squamous cell carcinoma
}

\author{
Joshua D. Smith BA ${ }^{1}$ | Andrew C. Birkeland MD ${ }^{1}$ | Andrew J. Rosko MD ${ }^{1}$ | Rebecca C. Hoesli MD ${ }^{1}$ | \\ Susan K. Foltin BS ${ }^{1}$ | Paul Swiecicki MD ${ }^{2,3} \mid$ Michelle Mierzwa MD ${ }^{2,4} \mid$ Steven B. Chinn MD $^{1}$ | \\ Andrew G. Shuman MD ${ }^{1}$ | Kelly M. Malloy MD ${ }^{1}$ | Keith A. Casper MD ${ }^{1}$ | Scott A. McLean MD ${ }^{1}$ | \\ Gregory T. Wolf MD ${ }^{1}$ | Carol R. Bradford MD ${ }^{1,2}$ | Mark E. Prince MD ${ }^{1,2}$ | \\ John Chad Brenner PhD ${ }^{1,2,5,6}$ (D) । Matthew E. Spector MD ${ }^{1,2}$
}

${ }^{1}$ Department of Otolaryngology - Head and Neck Surgery, University of Michigan Medical School, Ann Arbor, Michigan

${ }^{2}$ Rogel Comprehensive Cancer Center, University of Michigan Medical School, Ann Arbor, Michigan

${ }^{3}$ Department of Internal Medicine, Division of Hematology and Oncology, University of Michigan Medical School, Ann Arbor, Michigan

${ }^{4}$ Department of Radiation Oncology, University of Michigan Medical School, Ann Arbor, Michigan

${ }^{5}$ Program in Cellular and Molecular Biology, University of Michigan Medical School, Ann Arbor, Michigan

${ }^{6}$ Department of Pharmacology, University of Michigan Medical School, Ann Arbor, Michigan

\section{Correspondence}

Matthew E. Spector, MD, Department of Otolaryngology - Head and Neck Surgery, University of Michigan, 1500 E. Medical Center Dr., 1904 Taubman Center, Ann Arbor, MI 48109-5312.

Email: mspector@umich.edu

Chad Brenner, PhD, Department of Otolaryngology Head and Neck Surgery, University of Michigan, 1150 E. Medical Center Dr., 9301B MSRB3, Ann Arbor, MI 48109-0602.

Email: chadbren@umich.edu

Funding information

National Institutes of Health, Grant/Award Numbers: P30-CA046592R01-CA194536T32 DC535615U01-DE025184, R01-CA194536, P30-CA046592, U01-DE025184, T32 DC535615; American Head and Neck Society

\begin{abstract}
Background: We sought to describe targeted DNA sequencing data of persistent/ recurrent laryngeal squamous cell carcinoma (LSCC) and to compare gene-specific alteration frequencies with that of primary, untreated LSCC specimens from The Cancer Genome Atlas (TCGA).

Methods: The tumors of 21 patients with persistent/recurrent LSCC were subjected to targeted DNA sequencing using the Ion AmpliSeq Comprehensive Cancer Panel. Gene-specific alteration frequencies were compared (Chi-Square test) to primary, untreated LSCC sequencing data from TCGA using the cBioPortal platform. Results: Persistent/recurrent LSCC was characterized by a high rate of inactivating alterations in TP53 (38.1\%) and CDKN2A (33\%), amplification events of CCNDI (19.1\%), and ERBB2 (14.3\%), and NOTCH1 (19.1\%) mutations. Comparison of primary vs persistent/recurrent LSCC revealed significant differences in alteration frequencies of eight critical genes: BAP1, CDKN2A, DCUN1D1, MSH2, MTOR, PIK3CA, TET2, and TP53.
\end{abstract}

Conclusions: Our results provide preliminary support for a distinct mutational profile of persistent/recurrent LSCC that requires validation in larger cohorts.

\section{KEYWORDS}

laryngeal squamous cell carcinoma, larynx, persistent, recurrent, TCGA
This study was presented as a poster at the 2018 Multidisciplinary Head and Neck Cancers Symposium, Scottsdale, AZ (February 15-February 17, 2018).

\section{1 | INTRODUCTION}

Laryngeal squamous cell carcinoma (LSCC) remains a significant source of morbidity and mortality, with 4000 deaths annually in the United States. ${ }^{1}$ Organ preservation radiotherapy 
(RT) and chemoradiotherapy (CRT) have become standard of care for many primary LSCC. However, disease recurrence remains a difficult issue, with 5-year disease-free survival rates for advanced LSCC after initial RT or $\mathrm{CRT}^{2}$ ranging from 30 to $60 \% .{ }^{3,4}$ For patients with persistent/recurrent LSCC after initial treatment, prognosis is guarded. ${ }^{5-7}$ Our best data on the genomic landscape of LSCC comes from previously untreated patients within The Cancer Genome Atlas (TCGA). Thus, a better understanding of genetic drivers of persistent/recurrent LSCC to provide prognostic biomarkers and targets for novel therapeutics are needed in this patient cohort. ${ }^{8}$

Analysis of primary LSCC specimens in recent wholeexome sequencing (WES) studies by Stransky et al. $(n=15)^{9}$ and the Cancer Genome Atlas Network $(n=117)^{10}$ has identified high rates of TP53 mutations, activating PIK3CA alterations, $C C N D 1$ and EGFR amplification, $C D K N 2 A$ deletion, and NOTCH1 aberrations in primary LSCC. ERBB2 amplification has similarly emerged as a potential oncologic driver in a smaller subset of LSCC. ${ }^{11}$ Importantly, these studies did not specifically look into persistent/recurrent tumor mutational profiles after RT/CRT.

The biology of recurrent head and neck squamous cell carcinoma is not well characterized, though recent sequencing efforts have revealed unique, and occasionally targetable, mutational signatures in a handful of these tumors. ${ }^{12,13}$ There is significant rationale for distinct mutational signatures in recurrent LSCC given the poor initial clinical response of these tumors, the potential for de novo mutations due to RT/CRT, and tumor evolution and subclonal proliferation. As such, a better understanding of molecular alterations underlying these cancers is paramount to advancing precision oncology paradigms in this cohort.

We hypothesized that the mutational landscape in persistent/ recurrent LSCC will be distinct from primary, untreated LSCC. Herein, we report mutational profiles of persistent/recurrent LSCC in the largest patient cohort to date and illustrate important differences in mutational rates of LSCC driver genes by comparison to TCGA database of primary, untreated LSCC.

\section{2 | PATIENTS AND METHODS}

Our study used a convenient sample of patients with biopsyproven persistent/recurrent LSCC $(n=21)$ who were treated at the University of Michigan Hospital and Health Systems between 2000 and 2012. Clinical (eg, demographics, tobacco and alcohol history, tumor sub-site, American Joint Committee on Cancer [AJCC] stage, and treatment) and survival data were collected and summarized.

Formalin-fixed, paraffin-embedded (FFPE) specimens were obtained from the University of Michigan pathology archive under an Institutional Review Board (IRB)-approved protocol (HUM00080561). Tumor cores and adjacent normal tissue were taken from FFPE blocks, and genomic DNA was isolated using the Qiagen Allprep DNA/RNA FFPE kit
(Qiagen, Hilden, Germany) and quantified using a Qubit as previously described. ${ }^{14}$

Amplicon-based DNA sequencing of genes relevant to LSCC biology was performed on the Ion Torrent Personal Genome Machine, utilizing the Ion AmpliSeq Comprehensive Cancer Panel (Thermo Fisher Scientific, Waltham, Massachusetts), as previously described. ${ }^{11,15}$ Single-nucleotide variants (SNVs) and indels were called using the Torrent Variant Caller, annotated using ANNOVAR, and filtered for candidate non-synonymous somatic variants. ${ }^{16}$ Gene-level copy number variants $(\mathrm{CNV})$ were determined by taking the mean of the coverage-weighted per-probe ratios, as described. ${ }^{17}$ As per the manufacturer's specifications, the Ion AmpliSeq Comprehensive Cancer Panel yields $\geq 94 \%$ exome sequencing coverage at an average read depth of $350 \times{ }^{11,15}$

WES data of primary LSCC $(n=117)$ specimens was retrieved from the TCGA provisional dataset using the cBioPortal platform. ${ }^{18,19}$ Chi-Square test $(\alpha=0.05)$ using GraphPad Prism (GraphPad Software, La Jolla, California) was used for comparison of mutational rates in persistent/ recurrent vs primary LSCC.

\section{3 | RESULTS}

Clinical characteristics of the cohort are summarized in Table 1. All included patients were male with significant tobacco use history. The mean (range) age of included patients at diagnosis was 59 (40-77) years. Early- and latestage initial tumors were equally represented in our cohort. Primary treatment consisted of CRT $(n=10)$, RT $(n=9)$, and partial or total laryngectomy with adjuvant RT $(n=2)$. The mean (range) disease-free interval (DFI) was 29 (3-150) months. Sites of LSCC recurrence included local $(n=18)$, regional $(n=1)$, local and distant $(n=1)$, and distant $(n=1)$. Despite surgical salvage in $17(81 \%)$ patients, 9 (43\%) died of LSCC.

Targeted DNA sequencing of persistent/recurrent LSCC specimens revealed a high frequency of alterations also present in primary LSCC, including inactivating TP53 (38.1\%) and CDKN2A (33\%) mutations and complex NOTCH1 (19.1\%) alterations (Figure 1). Potentially targetable amplifications of CCNDI (19.1\%), ERBB2 (14.3\%), and PIK3CA $(9.5 \%)$ were also seen. Our modest sample size precludes definitive statistical analyses of the effect of specific genetic alterations on survival in our cohort. However, we are able to highlight mutational profiles of individual patients with persistent/recurrent LSCC who recurred and ultimately died of disease, thus nominating such genetic alterations for further study and potential therapeutic targeting.

Patient number 4 recurred locally 28 months after CRT for stage III primary disease, ultimately dying of recurrent LSCC 16 months after salvage laryngectomy. His recurrent tumor harbored missense mutations in BRCA2 and MSH2. Patient number 6 experienced a late, unresectable local 
TABLE 1 Clinical characteristics of patients with persistent/recurrent LSCC

\begin{tabular}{|c|c|c|c|c|c|c|c|c|c|c|c|}
\hline Patient no. & Sex & Age & $\begin{array}{l}\text { Smoking } \\
\text { status }\end{array}$ & $\begin{array}{l}\text { Pack } \\
\text { years }\end{array}$ & $\begin{array}{l}\text { Primary } \\
\text { stage }\end{array}$ & $\begin{array}{l}\text { Initial } \\
\text { treatment }\end{array}$ & $\begin{array}{l}\text { Recurrence } \\
\text { site }\end{array}$ & DFI (months) & $\begin{array}{l}\text { Recurrence } \\
\text { treatment }\end{array}$ & $\begin{array}{l}\text { Survival } \\
\text { months }^{\text {a }}\end{array}$ & $\begin{array}{l}\text { Died of } \\
\text { disease }\end{array}$ \\
\hline 1 & M & 72 & Former & 40 & III & CRT & Larynx & 10 & Surgery & 104 & No \\
\hline 2 & M & 48 & Current & 70 & IV & RT & Larynx & 4 & Surgery & 37 & No \\
\hline 3 & M & 64 & Former & 120 & II & CRT & Larynx & 21 & Surgery & 87 & No \\
\hline 4 & M & 62 & Former & 20 & III & CRT & Larynx & 28 & Surgery & 16 & Yes \\
\hline 5 & M & 59 & Current & 80 & IV & Surgery & Neoglottis, lung & 118 & Surgery $^{b}$ & 102 & No \\
\hline 6 & M & 68 & Former & 20 & IV & CRT & Larynx & 63 & None & 4 & Yes \\
\hline 7 & M & 65 & Former & 63 & I & RT & Larynx & 9 & Surgery & 98 & No \\
\hline 8 & M & 52 & Former & 50 & IV & CRT & Larynx & 10 & Surgery & 67 & Yes \\
\hline 9 & M & 68 & Current & 80 & II & CRT & Larynx & 4 & Surgery & 9 & No \\
\hline 10 & M & 58 & Current & 30 & I & RT & Larynx & 13 & Surgery & 12 & Yes \\
\hline 11 & M & 40 & Current & 20 & II & RT & Larynx & 71 & Surgery & 55 & No \\
\hline 12 & M & 52 & Current & 40 & IV & CRT & Tongue Base & 50 & Surgery & 7 & Yes \\
\hline 13 & M & 57 & Former & 40 & II & $\mathrm{RT}$ & Larynx & 3 & Surgery & 114 & No \\
\hline 14 & M & 61 & Current & 45 & IV & CRT & Diffuse & 4 & Palliative RT & 3 & Yes \\
\hline 15 & M & 63 & Former & 180 & IV & CRT & Larynx & 9 & Surgery & 80 & No \\
\hline 16 & M & 75 & Former & 30 & I & RT & Larynx & 16 & Surgery & 52 & No \\
\hline 17 & M & 50 & Former & 30 & III & RT & Larynx & 3 & Surgery & 10 & No \\
\hline 18 & M & 53 & Current & 35 & IV & CRT & Neck & 9 & Surgery & 6 & Yes \\
\hline 19 & M & 51 & Former & 35 & I & RT & Larynx & 10 & Surgery & 88 & No \\
\hline 20 & M & 43 & Current & 20 & II & Surgery & Larynx & 150 & RT & 44 & Yes \\
\hline 21 & M & 77 & Former & 30 & I & RT & Larynx & 8 & Surgery & 44 & Yes \\
\hline
\end{tabular}

Abbreviations: CRT, chemoradiotherapy; DFI, disease-free interval; LSCC, laryngeal squamous cell carcinoma; RT, radiotherapy.

a Time from date of recurrence to most recent clinical follow-up or death from any cause.

${ }^{\mathrm{b}}$ Patient underwent salvage laryngectomy and thoracoscopic wedge resection of solitary lung metastasis.

recurrence, 63 months after CRT for stage IV disease, and ultimately died 4 months later. His recurrent tumor was characterized by $C D K N 2 A$ deletion and amplification of PIK3CA and SOX2. Patient number 10 recurred locally 13 months after RT for stage I disease and died of recurrent disease 1 year after salvage laryngectomy. His recurrent LSCC harbored nonsense NOTCH1 alteration and TP53 missense mutation. Finally, patient number 14 recurred distantly 4 months after CRT for stage IV disease, dying 3 months after initiation of palliative RT. His tumor harbored multiple deleterious alterations, including inactivating missense mutations in $A T M$, NOTCHI, and $T P 53$, as well as $E R B B 2$ amplification and $C D K N 2 A$ deletion.

We next compared the alteration frequency of 52 genes in the Ion AmpliSeq Comprehensive Cancer Panel between our persistent/recurrent LSCC cohort and the primary, untreated TCGA cohort $(n=117)$ (Figure 2, Supporting Information Table S1). Primary LSCC harbored a significantly higher rate of characteristic CDKN2A, PIK3CA, and TP53 alterations. Amplification of $D C U N 1 D 1$, a candidate oncogene at the $3 \mathrm{q}$ chromosomal locus implicated in squamous cell carcinogenesis, was also seen more frequently in primary LSCC (Figure 2$).{ }^{20}$ Conversely, the PI3K pathway effector MTOR, the critical DNA mismatch repair gene $M S H 2$, and the $B R C A l$-associated protein $B A P 1$ harbored complex mutational patterns at a higher frequency in persistent/recurrent LSCC.

\section{4 | DISCUSSION}

Disease recurrence after definitive initial treatment for LSCC is a therapeutic challenge due to constraints imparted by previous therapies, considerable morbidity of salvage surgery, and potential acquired resistance of disease to chemotherapy and radiation. Prognosis for recurrent LSCC has stagnated in recent decades, with median survival after recurrence of 22 months for patients eligible for salvage surgery or reirradiation and less than 12 months in those administered palliative chemotherapy. ${ }^{21}$ The complex genetic alterations driving LSCC recurrence, progression, and treatment resistance need to be better characterized to guide development of precision therapeutics in the bench-to-bedside paradigm.

Biologically, cancer recurrence is preceded by a complex, branched evolution of subclonal populations within the primary tumor that persist under selective treatment pressures and propagate with further accumulation of deleterious mutations. ${ }^{22}$ Hedberg et al. performed WES of eight primary tumor-metachronous recurrence pairs of head and neck squamous cell carcinoma (HNSCC). ${ }^{12}$ The authors showed that nearly $40 \%$ of somatic variants identified in the recurrent cancers were new and absent in the primary tumor, including amplification of CCNDI and nonsynonymous mutations of $D D R 2$ (discoidin domain receptor tyrosine kinase 2). DDR2 


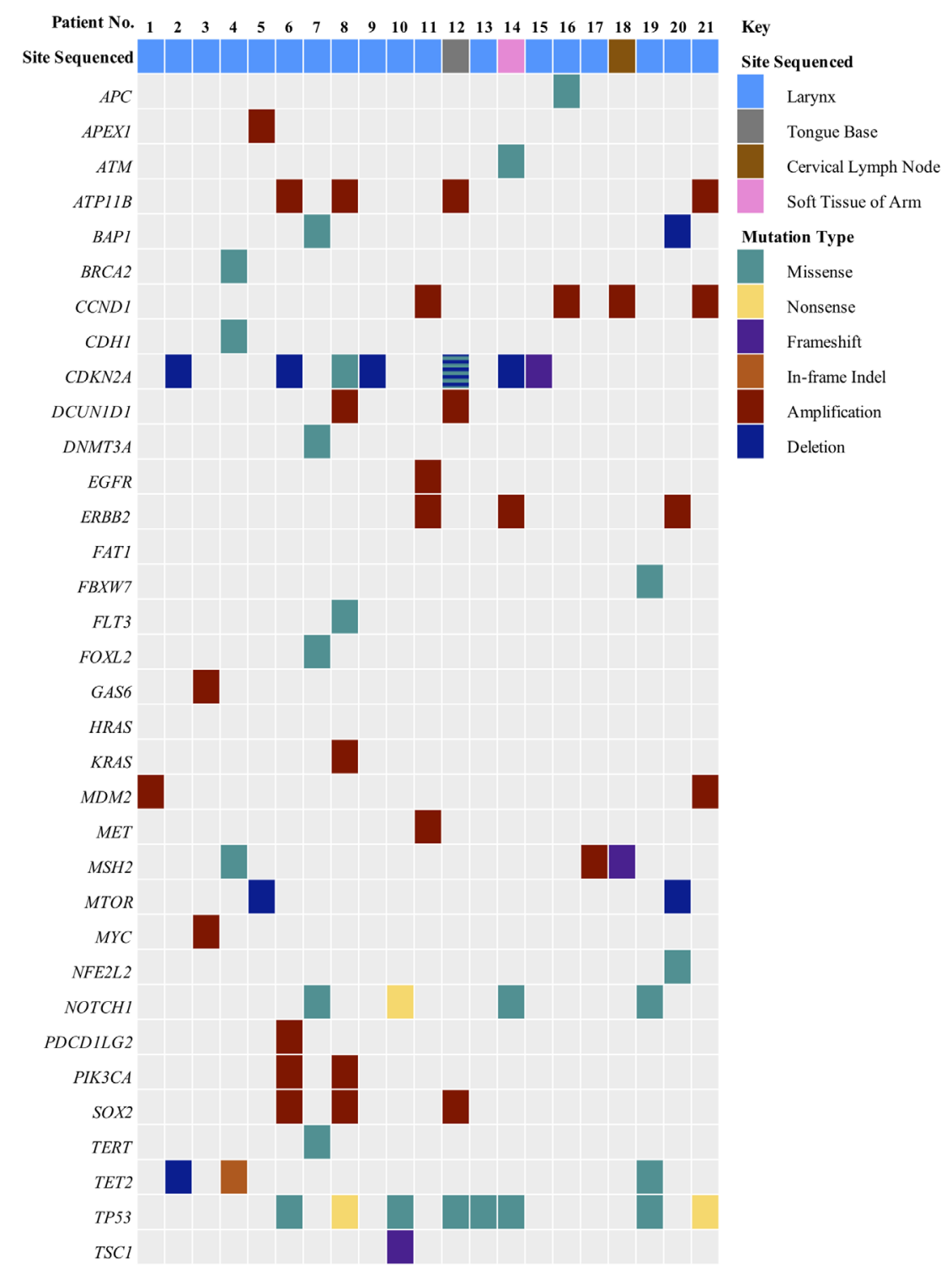

FIGURE 1 Mutational profiles of persistent/recurrent laryngeal squamous cell carcinoma (LSCC). Each column represents one patient/sequenced tumor. Genes altered in $\geq 1$ patient/sequenced tumor shown [Color figure can be viewed at wileyonlinelibrary.com]

alterations promote cellular migration, invasion, and metastasis in $\mathrm{HNSCC}^{23}$ and confer sensitivity to the SRC-family kinase inhibitor dasatinib in other cancers. ${ }^{24}$ Implementation of routine sequencing panels in persistent/recurrent LSCC management may therefore exploit unique biology of tumor recurrence and nominate novel therapeutic options in this patient cohort.

To date, ours is the largest targeted sequencing cohort of persistent/recurrent LSCC, allowing for comparison to primary LSCC mutational profiles and nomination of specific genetic aberrations for further study. Because of our modest sample size, we were unable to derive statistical models for predicting survival outcomes in persistent/recurrent LSCC on the basis of specific genetic perturbations. However, it is evident that the recurrent tumors of patients with particularly poor outcomes harbor multiple complex, and potentially targetable, genomic alterations that are attractive candidates for further study.

Surprisingly, we found higher rates of deleterious alterations in CDKN2A, PIK3CA, and TP53 in primary, untreated LSCC
(Figure 2). One possibility for this observation is the existence of a sampling bias within our cohort such that alteration frequencies for these crucial genes in persistent/recurrent LSCC are falsely lowered. These findings require validation in larger

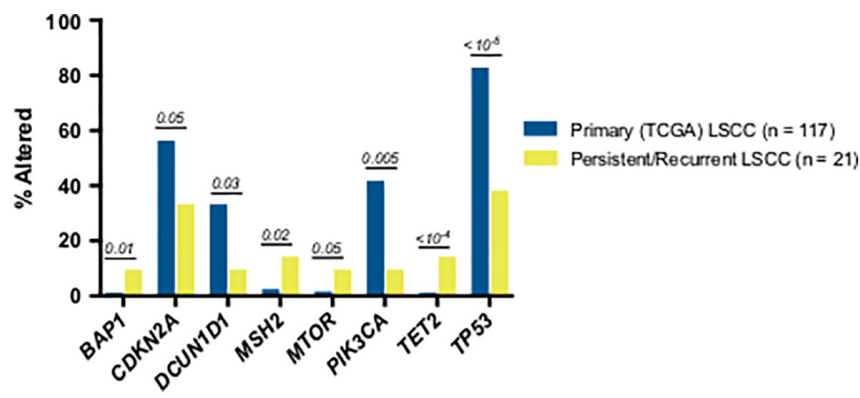

FIGURE 2 Frequency of alterations in eight critical genes differs in primary vs persistent/recurrent laryngeal squamous cell carcinoma (LSCC). $P$-value for comparison (Chi-square) listed above each gene [Color figure can be viewed at wileyonlinelibrary.com] 
sequencing cohorts. However, our preliminary data suggest that LSCC recurrence and progression may rely on more complex, alternative patterns of dysregulation in cellular growth and cellcycle control pathways relative to primary LSCC. This unique pathogenetic complexity of persistent/recurrent LSCC likely manifests clinically as resistance to conventional chemotherapy and targeted agents and disease progression and early mortality.

The histone modifier BAPI and the DNA mismatch repair gene $M S H 2$ were both more frequently altered in persistent/ recurrent LSCC. BAPI was recently shown to induce resistance to RT in HNSCC by modulating homologous recombination mechanisms. ${ }^{25}$ Defects in $\mathrm{MSH} 2$ promote microsatellite instability and accumulation of deleterious mutations by interference with nucleotide mismatch repair processes. ${ }^{26}$ Aberrations in cellular DNA damage repair pathways may therefore be central to pathogenesis of persistent/recurrent LSCC and are attractive targets for potential therapeutic modulation.

Ultimately, confirmation of unique mutational profiles of primary vs persistent/recurrent LSCC suggested by our study and other authors is needed in larger cohorts. Similarly, how genomic aberrations in LSCC interact with other aspects of tumor biology (eg, immune microenvironment) to promote disease recurrence and progression requires further elucidation to improve outcomes in this population. ${ }^{27}$

Our study has several limitations worth noting. Our persistent/recurrent LSCC samples were sequenced from FFPE on a targeted exome, commercially available sequencing platform. This is in contrast to TCGA, which used wholegenome sequencing and WES of fresh-frozen primary tumors. Our comparison of mutational rates in primary vs persistent/recurrent LSCC needs to be interpreted in this light. However, several recent studies have demonstrated high (ie, > 90\%) concordance rates in detection of SNVs and $\mathrm{CNV}$ s in coding genomic regions between matched freshfrozen and FFPE tumor samples. ${ }^{28-30}$ In addition, comparison of amplicon-based, targeted exome sequencing to TCGA dataset is a well-established practice in the HNSCC literature. $^{11,13}$ Thus, we believe that these differences in DNA sequencing methodology do not significantly diminish the validity of our comparisons and conclusions. Second, the relatively small number of persistent/recurrent LSCC samples in our study limits our ability to draw sophisticated statistical conclusions regarding the impact of specific genomic alterations on survival in persistent/recurrent LSCC. Finally, due to limited availability of archived tissue from our patients' primary tumors, we were unable to directly compare gene alteration frequencies in primary vs persistent/recurrent LSCC within individual patients.

Future studies should prioritize whole-exome and transcriptomic profiling of larger patient cohorts enriched for both locoregional and distant recurrences of LSCC to fully capture the unique mutational profiles of persistent/recurrent LSCC. As in Hedberg et al., ${ }^{12}$ continued efforts to characterize pathogenetic mechanisms of disease recurrence by DNA sequencing of matched primary and recurrent LSCC specimens from individual patients is paramount.

\section{I CONCLUSIONS}

Targeted DNA sequencing of persistent/recurrent LSCC reveals important differences in gene-specific alteration frequencies of these tumors compared to primary, untreated LSCC from TCGA. Our results provide preliminary support for a distinct mutational profile of persistent/recurrent LSCC that requires confirmation in larger cohorts. These data may be leveraged in future precision oncology initiatives to inform patient and mutation-centric targeted interventions in this patient population. ${ }^{31}$

\section{ACKNOWLEDGMENTS}

We thank C.J.L., D.H.H., A.K.C., and S.A.T. for technical support.

\section{ORCID}

John Chad Brenner (1) https://orcid.org/0000-0003-3238-1111

\section{REFERENCES}

1. Surveillance, Epidemiology, and End Results (SEER) Program - National Cancer Institute. Cancer Stat Facts: Laryngeal Cancer. 2016. Accessed February 6, 2018 from https://seer.cancer.gov/statfacts/html/laryn.html.

2. Department of Veterans Affairs Laryngeal Cancer Study Group, Wolf G, Fisher SG, et al. Induction chemotherapy plus radiation compared with surgery plus radiation in patients with advanced laryngeal cancer. $N$ Engl $J$ Med. 1991;324:1684-1690.

3. Forastiere AA, Zhang Q, Weber RS, et al. Long-term results of RTOG 91-11: a comparison of three nonsurgical treatment strategies to preserve the larynx in patients with locally advanced larynx cancer. J Clin Oncol. 2013; 31:845-852.

4. Denaro N, Russi EG, Lefebvre JL, Merlano MC. A systematic review of current and emerging approaches in the field of larynx preservation. Radiother Oncol. 2014;110:16-24

5. Birkeland AC, Beesley L, Bellile E, Rosko AJ, et al. Predictors of survival after total laryngectomy for recurrent/persistent laryngeal squamous cell carcinoma. Head Neck. 2017;39:2512-2518.

6. Brandstorp-Boesen J, Sorum Falk R, Boysen M, Brondbo K. Impact of stage, management and recurrence on survival rates in laryngeal cancer. PLoS One. 2017;12:e179371.

7. Forastiere AA, Weber RS, Trotti A. Organ preservation for advanced larynx cancer: issues and outcomes. J Clin Oncol. 2015;33:3262-3268.

8. Ludwig ML, Birkeland AC, Hoesli R, Swiecicki P, Spector ME, Brenner JC. Changing the paradigm: the potential for targeted therapy in laryngeal squamous cell carcinoma. Cancer Biol Med. 2016;13:87-100.

9. Stransky N, Egloff AM, Tward AD, et al. The mutational landscape of head and neck squamous cell carcinoma. Science. 2011;333:1157-1160.

10. The Cancer Genome Atlas Network. Comprehensive genomic characterization of head and neck squamous cell carcinomas. Nature. 2015;517: 576-582.

11. Birkeland AC, Yanik M, Tillman $\mathrm{BN}$, et al. Identification of targetable ERBB2 aberrations in head and neck squamous cell carcinoma. JAMA Otolaryngol Head Neck Surg. 2016;142:559-567.

12. Hedberg ML, Goh G, Chiosea SI, et al. Genetic landscape of metastatic and recurrent head and neck squamous cell carcinoma. J Clin Invest. 2016;126: 169-180.

13. Morris LG, Chandramohan R, West $\mathrm{L}$, et al. The molecular landscape of recurrent and metastatic head and neck cancers: insights from a precision oncology sequencing platform. JAMA Oncol. 2017;3:244-255. 
14. Tillman BN, Yanik M, Birkeland AC, et al. Targeted sequencing of an epidemiologically low risk patient defines fibroblast growth factor family aberrations as a putative driver of head and neck squamous cell carcinoma. Head Neck. 2016;38:1646-1652.

15. Warrick JI, Hovelson DH, Amin A, et al. Tumor evolution and progression in multifocal and paired non-invasive/invasive urothelial carcinoma. Virchows Arch. 2015;466:297-311.

16. Wang $\mathrm{K}, \mathrm{Li} \mathrm{M}$, Hakonarson $\mathrm{H}$. ANNOVAR: functional annotation of genetic variants from high-throughput sequencing data. Nucleic Acids Res. 2010;38:e164.

17. Grasso $\mathrm{C}$, Butler $\mathrm{T}$, Rhodes $\mathrm{K}$, et al. Assessing copy number alterations in targeted, amplicon-based next-generation sequencing data. J Mol Diagn. 2015; $17: 53-63$

18. Cerami E, Gao J, Dogrusoz A, et al. The cBio cancer genomics portal: an open platform for multidimensional cancer genomics data. Cancer Discov. 2012;2:401-404.

19. Gao J, Aksoy BA, Dogrusoz U, et al. Integrative analysis of complex cancer genomics and clinical profiles using the cBioPortal. Sci Signal. 2013; 6(269):pl1.

20. Sarkaria I, O-charoenrat $P$, Talbot SG, et al. Squamous cell carcinoma related oncogene/DCUN1D1 is highly conserved and activated by amplification in squamous cell carcinomas. Cancer Res. 2006;66:9437-9444.

21. Ho AS, Kraus DH, Ganly I, Lee NY, Shah JP, Morris LG. Decision making in the management of recurrent head and neck cancer. Head Neck. 2014; 36(1):144-151.

22. Gerlinger $M$, Rowan AJ, Horswell $S$, et al. Intratumor heterogeneity and branched evolution revealed by multiregion sequencing. $N$ Engl J Med. 2012;366:883-892.

23. $\mathrm{Xu} \mathrm{J}, \mathrm{Lu} \mathrm{W}$, Zhang $\mathrm{S}$, et al. Overexpression of DDR2 contributes to cell invasion and migration in head and neck squamous cell carcinoma. Cancer Biol Ther. 2014;15(5):612-622.

24. Hammerman PS, Sos ML, Ramos AH, et al. Mutations in the DDR2 kinase gene identify a novel therapeutic target in squamous cell lung cancer. Cancer Discov. 2011;1:78-89.
25. Liu X, Kumar M, Yang L, et al. BAP1 is a novel target in HPV-negative head and neck cancer. Clin Cancer Res. 2018;24(3):600-607.

26. Cortes-Ciriano I, Lee S, Park W, Kim T, Park P. A molecular portrait of microsatellite instability across multiple cancers. Nat Commun. 2017;8:15180.

27. Hoesli R, Birkeland AC, Rosko AJ, et al. Proportion of CD4 and CD8 tumor infiltrating lymphocytes predicts survival in persistent/recurrent laryngeal squamous cell carcinoma. Oral Oncol. 2018;77:83-89.

28. Robbe $\mathrm{P}$, Popitsch $\mathrm{N}$, Knight $\mathrm{S}$, et al. Clinical whole-genome sequencing from routine formalin-fixed, paraffin-embedded specimens: pilot study for the 100,000 genomes project. Genet Med. 2018. Epub ahead of print 2018 Feb 1. https://doi.org/10.1038/gim.2017.241.

29. Hedegaard $\mathrm{J}$, Thorsen $\mathrm{K}$, Lund $\mathrm{M}$, et al. Next-generation sequencing of RNA and DNA isolated from paired fresh-frozen and formalin-fixed paraffin-embedded samples of human cancer and normal tissue. PLoS One. 2014;9(5):e98187.

30. Oh E, Choi Y, Kwon M, et al. Comparison of accuracy of whole-exome sequencing with formalin-fixed paraffin-embedded and fresh frozen tissue samples. PLoS One. 2015;10(12):e0144162.

31. Birkeland AC, Uhlmann WR, Brenner JC, Shuman AG. Getting personal: head and neck cancer management in the era of genomic medicine. Head Neck. 2016;38(S1):e2250-e2258.

\section{SUPPORTING INFORMATION}

Additional supporting information may be found online in the Supporting Information section at the end of the article.

How to cite this article: Smith JD, Birkeland AC, Rosko AJ, et al. Mutational profiles of persistent/ recurrent laryngeal squamous cell carcinoma. Head \& Neck. 2019;41:423-428. https://doi.org/10.1002/hed. 25444 